Check for updates

Cite this: RSC Adv., 2017, 7, 49649

Received 11th October 2017

Accepted 18th October 2017

DOI: $10.1039 / c 7 r a 11186 j$

rsc.li/rsc-advances

\section{A dual-functional surface with hierarchical micro/ nanostructure arrays for self-cleaning and antireflection}

\author{
Zhenwei Mao, ${ }^{a}$ Wei Cao, ${ }^{a}$ Jie Hu, (D) *a Lan Jiang, ${ }^{a}$ Andong Wang, ${ }^{a}$ Xin $\mathrm{Li},{ }^{a}$ Jing $\mathrm{CaO}^{a}$ \\ and Yongfeng Lu (iD ${ }^{b}$
}

Surfaces with hierarchical micro/nanostructures have been widely fabricated for their extensive applications in self-cleaning, antireflection, etc. However, a flexible and highly efficient method for obtaining such surfaces remains a great challenge, especially for metals. In this paper, we propose a simple and tunable approach for fabricating a dual-functional surface with patterned hierarchical micro/nanostructure arrays. A femtosecond laser was used to remove the $\mathrm{SiO}_{2}$ thin film, coated on the $\mathrm{Cu}$ substrate, masklessly and selectively. Then the arrays of micro-protrusions decorated with nanoneedles were synthesized successfully through thermal oxidation. We demonstrate that the morphology of the microprotrusions remarkably affects the surface properties, which can be flexibly tuned by controlling femtosecond laser parameters. By optimizing the laser parameters (e.g. scanning mode and laser fluence), we can achieve high performances with respect to superhydrophobicity, with a maximum contact angle of $161^{\circ}$ and extremely low adhesion with a minimum sliding angle of less than $1.7^{\circ}$, thus demonstrating its self-cleaning function. In addition, antireflection properties with a minimum reflectance of less than $1 \%$ at a wavelength range of $700-800 \mathrm{~nm}$ can be achieved and the total reflectance can be steadily below $6 \%$ over a broad wavelength range of $600-1150 \mathrm{~nm}$.

\section{Introduction}

Over the past decades, micro/nanostructures have received considerable research interest because of their extensive applications in self-cleaning, ${ }^{1-6}$ oil-water separation, ${ }^{2,7,8}$ anticorrosion, ${ }^{3,4,9}$ optical enhancement, ${ }^{5,10-12}$ water splitting, ${ }^{13}$ catalyst, ${ }^{14-16}$ antireflection, ${ }^{17,18}$ and surface-enhanced Raman spectroscopy detection., ${ }^{3,6,19-21}$ There are plenty of hierarchical micro/nanostructure surfaces which have been inspired by nature. ${ }^{22-26}$ Jiang et al. reviewed the designs of bioinspired multifunctional materials ${ }^{27}$ and indicated that biological surfaces have some unique properties e.g. wettability, mechanical properties, structure color, and optical properties. The wettability of superhydrophobic surfaces with a static contact angle (CA) greater than $150^{\circ}$ and a sliding angle less than $10^{\circ}$ is mainly determined by the chemical composition, surface roughness, and geometric morphology. ${ }^{28}$ In particular, surfaces bioinspired by the lotus leaf effect ${ }^{29,30}$ have been increasingly researched because of their superhydrophobicity. The superhydrophobicity of lotus leaves mainly depends on the hierarchical micro/nanostructures rather than the effect of the

${ }^{a}$ Laser Micro/Nano Fabrication Laboratory, School of Mechanical Engineering, Beijing Institute of Technology, Beijing, 100081, P. R. China.E-mail: jiehu2@bit.edu.cn

${ }^{b}$ Department of Electrical Engineering, University of Nebraska-Lincoln, Lincoln, $N E$ 68588-0511, USA plant epicuticular wax that provides a primary waterproof barrier and protection against different environments. To obtain the analogy of superhydrophobicity surfaces with respect to the lotus leaf effect, it is necessary to fabricate hierarchical micro/nanostructures.

Recently, many hierarchical micro/nanostructures have been fabricated using conventional methods such as electrochemical processes,${ }^{31}$ lithography, ${ }^{32}$ and galvanic replacement reaction. ${ }^{33}$ For example, Yang et al. shown that the morphology of superhydrophobicity surfaces can be changed from cauliflower-like to dendritic-like through a galvanic replacement reaction with different concentrations of $\mathrm{AgNO}_{3}$ solution. ${ }^{33}$ However, this reaction in solution is less controllable and cannot achieve patterned micro/nano-structures. Zhang et al. reported heterohierarchical micro/nanostructures on $\mathrm{Cu}$ foils with a $\mathrm{CA}$ of $165^{\circ}$ and a sliding angle of less than $2^{\circ}$ using photolithography coupled with electrochemical deposition. ${ }^{3}$ Although patterned micro/nanostructures have been achieved using this method to obtain superhydrophobicity surfaces, the photomasks used in photolithography are complicated and expensive, ${ }^{34}$ limiting their further industrial applications. Femtosecond laser processing is a one-step direct maskless fabrication technique and has intrinsic advantages over traditional methods with respect to controllability and economy. ${ }^{35,36}$

In this paper, we propose a simple and tunable approach for fabricating a dual-functional surface with patterned 
a)
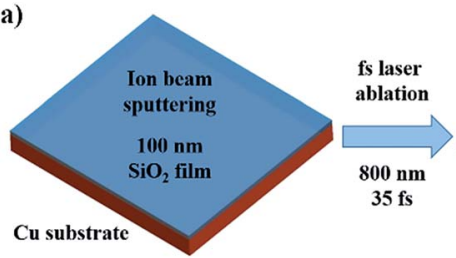

b)

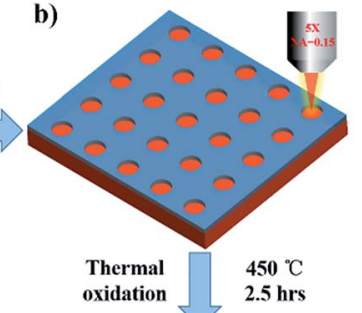

oxidation c)

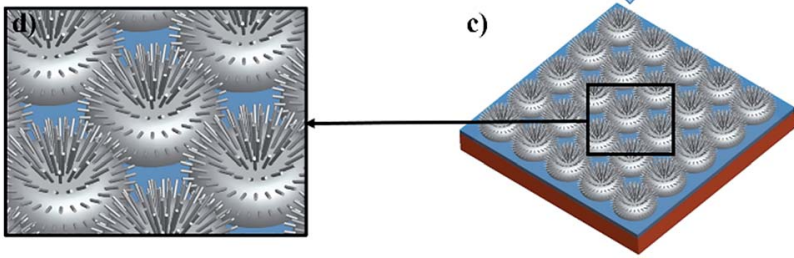

Fig. 1 Schematic procedures of the micro/nanostructures fabrication. (a) Prepared $\mathrm{Cu}$ substrate with $\mathrm{SiO}_{2}$ film coated by ion beam sputtering. (b) Removal of $\mathrm{SiO}_{2}$ thin film using femtosecond laser. (c) $\mathrm{CuO}$ micro/nanostructure growing on substrate after thermal oxidation. (d) Magnified structure units.

hierarchical micro/nanostructure arrays. The fabrication process is illustrated in Fig. 1. First, the $\mathrm{Cu}$ substrate was coated with a $\mathrm{SiO}_{2}$ film through ion beam sputtering. Subsequently, a uniform dot or line array was fabricated using a femtosecond laser. Finally, an array of micro-protrusions decorated with $\mathrm{CuO}$ nanoneedles was generated using thermal oxidation in a muffle furnace. The prepared hierarchical micro/nanostructures were $\mathrm{CuO}$ micro-protrusions decorated with $\mathrm{CuO}$ nanoneedles, exhibiting excellent superhydrophobicity and antireflection properties over a broad range of wavelengths.

\section{Experimental}

\section{Materials}

Polished $\mathrm{Cu}$ substrates $(10 \mathrm{~mm} \times 10 \mathrm{~mm} \times 1 \mathrm{~mm}, 99.9999 \%$ purity) were purchased from Hefei Kejing Materials Technology Co., Ltd. The $\mathrm{Cu}$ substrates were coated with a $100 \mathrm{~nm}$-thick $\mathrm{SiO}_{2}$ film through ion beam sputtering.

\section{Laser ablation of the $\mathrm{SiO}_{2}$ thin film on a $\mathrm{Cu}$ substrate}

The patterned dot and line arrays were fabricated using a modelocked Ti: sapphire laser system (Spectra Physics, Inc., Santa Clara, California, USA; $800 \mathrm{~nm}, 35 \mathrm{fs}, 1 \mathrm{kHz}$ ). The Gaussian profile laser beam was focused perpendicular to the substrate by an objective lens (Olympus MPLFLN $5 \times$; numerical aperture $[\mathrm{NA}]=0.15)$. The $\mathrm{Cu}$ sample was mounted on a six-axis motion stage (M-840.5DG, PI, Inc., Karlsruhe, Germany) with a resolution of $1 \mu \mathrm{m}$ in the $X Y$ directions and $0.5 \mu \mathrm{m}$ in the $Z$ direction. The motion stage was computer-controlled to efficiently fabricate the dot and line array structure. The laser fluence was adjusted from 0.9 to $10.5 \mathrm{~J} \mathrm{~cm}^{-2}$ while fabricating the dot array, and the laser fluence was adjusted from 0.54 to $1.2 \mathrm{~J} \mathrm{~cm}^{-2}$ while fabricating the line array. The dot-to-dot spacing was $15 \mu \mathrm{m}$ and the line-to-line spacing was $10 \mu \mathrm{m}$. After laser ablation, all the samples were cleaned ultrasonically with deionized water and alcohol for $5 \mathrm{~min}$ to remove debris.

\section{Thermal oxidation}

After the laser ablation, the samples were heated at $450{ }^{\circ} \mathrm{C}$ for $2.5 \mathrm{~h}$ in a muffle furnace (GHA12/300, Carbolite) to grow $\mathrm{CuO}$ micro-protrusions decorated with $\mathrm{CuO}$ nanoneedles. Then, the samples were cooled to room temperature.

\section{Characterization}

The sample surface was characterized using a scanning electron microscope (SEM, FEI XL30 S-FEG). The composition of the hierarchical micro/nanostructures was analyzed through energy dispersive X-ray spectroscopy (EDX). The surface water contact angles were measured using an optical contact angle measuring system (OCA 15 Pro, Dataphysics Inc.) and SCA 20 measuring software through a sessile drop technique. Reflectance was measured through Fourier-transform infrared spectroscopy (FTIR, Vertex 70, Bruker) using the polished $\mathrm{Cu}$ surface as the background signal and the fabricated surface as the sample signal.

\section{Results and discussion}

\section{Microdot arrays fabricated using femtosecond laser}

The laser ablation of $\mathrm{SiO}_{2}$ thin films coated on bulk materials has been a topic of considerable researches in recent years. ${ }^{37-41}$ Most previous studies have focused on $\mathrm{SiO}_{2}$ films on $\mathrm{Si}$ substrates, and the mechanism of film ablation has typically been explained by a model of melting and vaporization. ${ }^{37}$ The ablation of $\mathrm{a} \mathrm{SiO}_{2}$ thin film on a $\mathrm{Cu}$ substrate could be understood to be a similar process. The results of a microdot array fabricated using a femtosecond laser are shown in Fig. 2. Fig. 2(a) shows an SEM image of the patterned microdot array fabricated through femtosecond laser ablation of the $\mathrm{SiO}_{2}$ film coated on the $\mathrm{Cu}$ substrate. The patterned microdot array could be achieved by femtosecond laser selective single-pulse ablation. The magnified SEM image of the ablated craters is shown in the insets of Fig. 2(b). The $\mathrm{SiO}_{2}$ film was completely removed through femtosecond laser ablation while the Cu substrate was almost undamaged, which is similar to a previous report on femtosecond laser ablation of a $\mathrm{SiO}_{2}$ film on a Si substrate. ${ }^{39}$ Moreover, the microdots exhibited an elliptical geometric
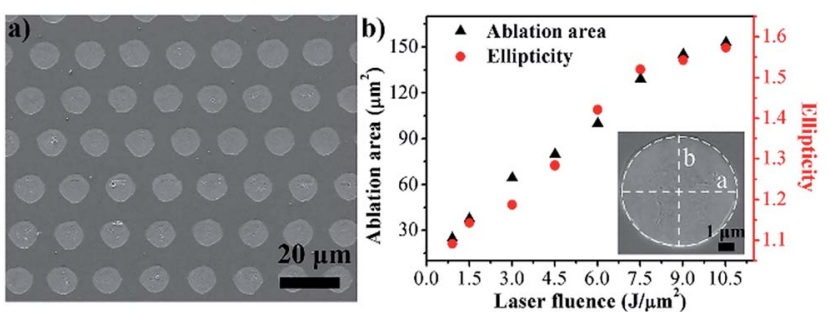

Fig. 2 (a) SEM image of the dot array fabricated through femtosecond laser ablation of $\mathrm{SiO}_{2}$ film coated on the Cu substrate (laser repetition: $100 \mathrm{~Hz}$; scanning speed: $1500 \mu \mathrm{m} \mathrm{s}^{-1}$; laser fluence: $1.5 \mathrm{~J} \mathrm{~cm}^{-2}$ ). (b) Dependence of ablation area and ellipticity on the laser fluence. The laser fluences were varied from 0.9 to $10.5 \mathrm{~J} \mathrm{~cm}^{-2}$. The inset is an SEM image of a microdot marked by dash lines, which represent the ablation area, the major axis, and the minor axis, respectively. 
morphology, wherein the major axis was parallel to the direction of laser polarization. A similar phenomenon has been reported in previous studies on femtosecond laser ablation of $\mathrm{Si}$ and $\mathrm{Cu}^{42,43}$ To qualitatively depict the aforementioned ellipticity of the ablated microdots, we defined parameter ellipticity as the aspect ratio of $a$ to $b$, where $a$ and $b$ represent the lengths of the major axis and minor axis, respectively. The dependence of the ablation area and ellipticity on the laser fluence is shown in Fig. 2(b). The ablation area of the $\mathrm{SiO}_{2}$ film increased as the laser fluence increased. That is, the size of the microdots can be controlled by adjusting the laser fluence. However, the laser fluence should not exceed certain threshold in order to prevent the $\mathrm{Cu}$ substrate from damage. Similarly, the ellipticity also increased with an increase in the laser fluence, as shown in Fig. 2(b). To explain this polarization-dependence phenomenon, anisotropic energy absorption should be considered. The energy of a polarized femtosecond laser is absorbed more efficiently along the laser polarization direction, as described by Fresnel coefficients. ${ }^{44}$ This anisotropic energy absorption effect facilitates the preferred ablation along the laser polarization.

\section{Thermal oxidation of the dot array}

$\mathrm{CuO}$ nanoneedles can be easily synthesized by heating a $\mathrm{Cu}$ substrate. ${ }^{46}$ Herein, after femtosecond laser ablation of the $\mathrm{SiO}_{2}$ thin film, the samples were placed in a muffle furnace to induce thermal oxidation. The parameters of thermal oxidation are important to obtain uniform micro-protrusions decorated with nanoneedles. In this study, all the samples were heated at

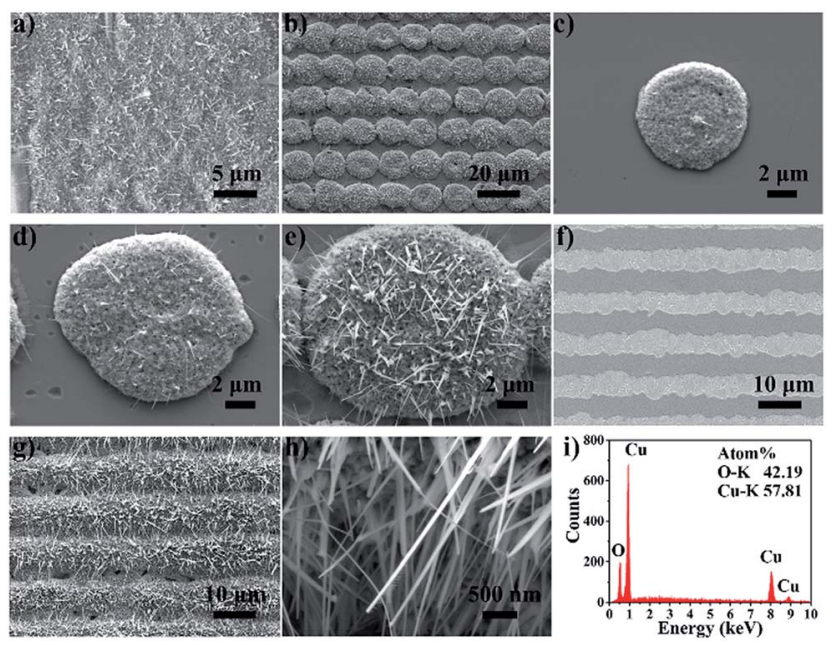

Fig. 3 (a) SEM image of $\mathrm{CuO}$ nanoneedles formed on bare Cu surface after thermal oxidation. The thermal oxidation lasted for $2.5 \mathrm{~h}$ at $450{ }^{\circ} \mathrm{C}$. (b) SEM image of microdot array patterned through femtosecond laser ablation and thermal oxidation when the was $6.0 \mathrm{~J} \mathrm{~cm}^{-2}$. (c-e) SEM images of micro-protrusion decorated with $\mathrm{CuO}$ nanoneedles after femtosecond laser ablation and thermal oxidation at different laser fluences: (c) 1.5, (d) 3.0 , and (e) $6.0 \mathrm{~J} \mathrm{~cm}^{-2}$. (f) SEM image of the microline array after femtosecond laser ablation of the $\mathrm{SiO}_{2}$ film (laser repetition: $1000 \mathrm{~Hz}$; scanning speed: $1000 \mathrm{\mu m} \mathrm{s}^{-1}$; laser fluence: $0.60 \mathrm{~J} \mathrm{~cm}^{-2}$ ). (g) SEM image of microridges decorated with $\mathrm{CuO}$ nanoneedles after femtosecond laser ablation and thermal oxidation. (h) Detailed view of the nanoneedles. (i) EDX result of the hierarchical line array.
$450{ }^{\circ} \mathrm{C}$ for $2.5 \mathrm{~h}$. Fig. 3(a) shows the SEM image of $\mathrm{CuO}$ nanoneedles formed on a bare $\mathrm{Cu}$ surface after thermal oxidation. The $\mathrm{Cu}$ surface was completely covered with $\mathrm{CuO}$ nanoneedles after thermal oxidation, but the resulting surface was inhomogeneous in space, which is consistent with a previous report. ${ }^{46}$ Fig. 3(b) shows the SEM image of a microdot array patterned through femtosecond laser ablation and thermal oxidation. The laser fluence of the femtosecond laser ablation was $6 \mathrm{~J} \mathrm{~cm}^{-2}$. After selective ablation of the $\mathrm{SiO}_{2}$ film, the $\mathrm{Cu}$ substrate was exposed to air. After thermal oxidation, the exposed $\mathrm{Cu}$ was oxidized to form $\mathrm{CuO}$ micro-protrusions decorated with nanoneedles, as shown in Fig. 3(b). The effects of the femtosecond laser fluences used in the ablation of the $\mathrm{SiO}_{2}$ film are illustrated in Fig. 3(c-e). Few $\mathrm{CuO}$ nanoneedles were formed on the micro-protrusion at $1.5 \mathrm{~J} \mathrm{~cm}^{-2}$ (Fig. 3(c)). However, when the laser fluence was increased to $3.0 \mathrm{~J} \mathrm{~cm}^{-2}$, some $\mathrm{CuO}$ nanoneedles were generated on the edge of the micro-protrusions (Fig. 3(d)). Abundant $\mathrm{CuO}$ nanoneedles were fabricated on the micro-protrusion at $6.0 \mathrm{~J} \mathrm{~cm}^{-2}$ (Fig. 3(e)). Moreover, the size of the micro-protrusions apparently increased with an increase of the laser fluence. Therefore, the femtosecond laser fluence considerably affects the subsequent growth performance of the thermal oxidation process. That is, the size of the microprotrusions and the morphology of the $\mathrm{CuO}$ nanoneedles can be adequately controlled using tunable femtosecond laser fluence.

\section{Results of the line array}

Femtosecond laser direct writing is a maskless technique for fabricating complicated structures with arbitrary shapes through programmed control. Except for the dot array, the line array can also be fabricated through femtosecond laser direct writing. Fig. 3(f) shows the SEM image of the microline array after femtosecond laser ablation of the $\mathrm{SiO}_{2}$ film. The edge of the ablated $\mathrm{SiO}_{2}$ film was slightly irregular because of the vibration of the motion stage. After thermal oxidation, the exposed $\mathrm{Cu}$ surface was oxidized to form $\mathrm{CuO}$ microridges decorated with abundant $\mathrm{CuO}$ nanoneedles (Fig. 3(g)). A further magnified SEM image shows that the diameters of certain nanoneedles were $55 \mathrm{~nm}$ and $23 \mathrm{~nm}$ (Fig. 3(h)). The EDX result in Fig. 3(i) indicates that the hierarchical line array comprised $\mathrm{Cu}$ and $\mathrm{O}$ elements. The chemical reactions involved in the formation of $\mathrm{CuO}$ can be given as follows: ${ }^{45,47,48}$

$$
\begin{gathered}
4 \mathrm{Cu}+\mathrm{O}_{2} \rightarrow 2 \mathrm{Cu}_{2} \mathrm{O} \\
2 \mathrm{Cu}_{2} \mathrm{O}+\mathrm{O}_{2} \rightarrow 4 \mathrm{CuO}
\end{gathered}
$$

\section{Wetting properties}

The wettability of the aforementioned prepared samples was further explored by testing the water contact angle (CA) in air. Fig. 4 illustrates the water CAs of hierarchical dot array and line array structures fabricated at different laser fluences before and after thermal oxidation. Before thermal oxidation, the CA of the dot array decreased from $99.2^{\circ}$ to $48.9^{\circ}$ as the laser fluences 

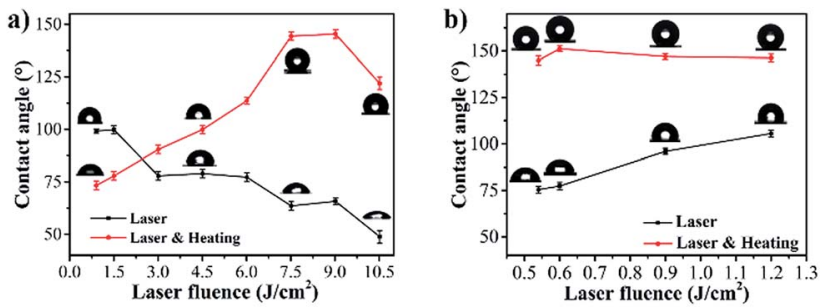

Fig. 4 Water contact angles of (a) hierarchical dot array and (b) hierarchical line array structures fabricated by different pulse energies before and after thermal oxidation.

increased from 0.9 to $10.5 \mathrm{~J} \mathrm{~cm}^{-2}$, exhibiting increasing hydrophilicity (Fig. 4(a), black line). However, after thermal oxidation, the CA of the dot array initially increased with the laser fluence and then decreased as the laser fluence exceeded $9.0 \mathrm{~J} \mathrm{~cm}^{-2}$ (Fig. 4(a), red line). The maximum CA could be $145.4^{\circ}$, indicating the hydrophobic property. Regarding the line array structure, the CA increased from $75.5^{\circ}$ to $105.6^{\circ}$ as the laser fluence increased from 0.54 to $1.2 \mathrm{~J} \mathrm{~cm}^{-2}$ before thermal oxidation, exhibiting increasing hydrophobicity (Fig. 4(b), black line). After thermal oxidation, the CA of the line array initially increased with the laser fluence and then decreased as the laser fluence exceeded $0.6 \mathrm{~J} \mathrm{~cm}^{-2}$ (Fig. 4(b), red line). The maximum CA could be $152^{\circ}$ without any chemical modification when the laser fluence was $0.6 \mathrm{~J} \mathrm{~cm}^{-2}$, indicating superhydrophobicity. It is worth noting that the $\mathrm{Cu}$ substrate would show severe damage when the laser fluence is higher than $1.2 \mathrm{~J} \mathrm{~cm}^{-2}$.

Fig. 5(a) displays a comparison of the water CA for different surfaces. As illustrated in Fig. 5(a), the bare $\mathrm{Cu}$ substrate (Cu-I) was slightly hydrophobic with a CA of $100^{\circ}$. When subjected to thermal oxidation, the smooth $\mathrm{Cu}$ surface was oxidized into abundant $\mathrm{CuO}$ nanoneedles, corresponding to Fig. 3(a). Because of the existence of $\mathrm{CuO}$ nanoneedles, the $\mathrm{CA}$ of the $\mathrm{CuO}$ surface (CuO-I) increased to $120^{\circ}$. When the $\mathrm{Cu}$ substrate was coated with a $100 \mathrm{~nm}$-thick $\mathrm{SiO}_{2}$ thin film (Cu-II), the CA was

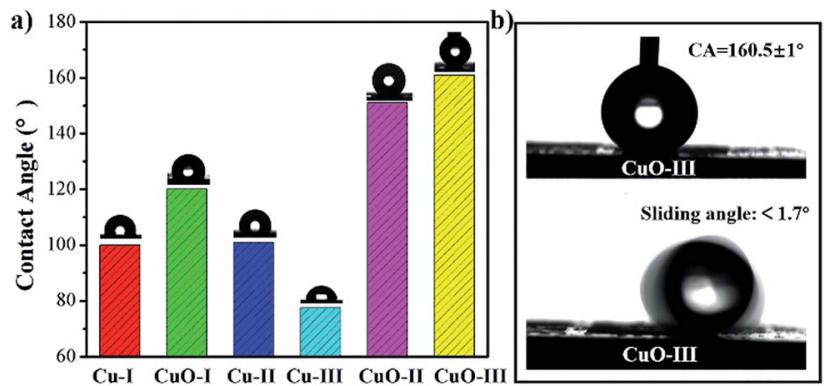

Fig. 5 (a) Comparison of the water contact angle on different surfaces. $\mathrm{Cu}-\mathrm{I}$ represent bare $\mathrm{Cu}$ surface. $\mathrm{CuO}-\mathrm{I}$ represents bare $\mathrm{Cu}$ surface after thermal oxidation. $\mathrm{Cu}-\mathrm{Il}$ represents bare $\mathrm{Cu}$ surface

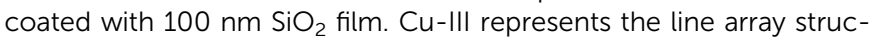
tures fabricated by fs laser at the fluence of $0.60 \mathrm{~J} \mathrm{~cm}^{-2}$ before thermal oxidation. CuO-II represents line array structures fabricated by fs laser at the fluence of $0.60 \mathrm{~J} \mathrm{~cm}^{-2}$ after thermal oxidation. CuO-III represents line array structures fabricated by fs laser at the fluence of $0.60 \mathrm{~J} \mathrm{~cm}^{-2}$ after thermal oxidation and chemical modification. (b) Contact angle and sliding angle of $\mathrm{CuO}-\mathrm{III}$. approximately $101^{\circ}$ and remained almost unchanged compared with $\mathrm{Cu}-\mathrm{I}$. When irradiated by femtosecond laser, the $\mathrm{SiO}_{2}$ film was selectively ablated. The CA of the line array structures $\left(\mathrm{Cu}^{-}\right.$ III) was approximately $77.4^{\circ}$, indicating a decrease in CA after femtosecond laser ablation of the $\mathrm{SiO}_{2}$ film at the fluence of 0.60 $\mathrm{J} \mathrm{cm}^{-2}$. However, after the thermal oxidation process, the CA of the line array (CuO-II) substantially increased to $152^{\circ}$, exhibiting the superhydrophobicity of this surface without any chemical modification. In other words, the wetting properties of the surface can be controlled from hydrophilicity to superhydrophobicity by the thermal oxidation process. To further improve the wetting properties, the chemical modification of the thermal-oxidized line array (CuO-II) could be achieved by silanization. After chemical modification, the CA of the surface (CuO-III) was approximately $160.5^{\circ}$. For a superhydrophobic surface, the sliding angle can reflect the adhesive force of water on the surface, which is defined as the critical angle at which the water begins to roll off the inclined plate. As shown in Fig. 5(b), the sliding angle of CuO-III was less than $1.7^{\circ}$, indicating that the water droplet could not adhere well on this superhydrophobic surface. Thus, the CuO-III surface was determined to have the best superhydrophobicity and lowest adhesion among all the fabricated surfaces.

\section{Self-cleaning}

A hierarchical micro/nanostructure line array usually has the property of self-cleaning due to its superhydrophobicity and low adhesion. To demonstrate the self-cleaning behaviour of different surfaces, sand particles were covered on the surfaces as contaminants. The process of a series of water droplets dripped on the CuO-III surface is shown in Fig. 6(a-c). The water droplets rolled off quickly and removed the contaminants on the CuO-III surface, exhibiting the considerable self-cleaning effect of the surface. The process of a series of water droplets dripped on the bare $\mathrm{Cu}$ surface is shown in Fig. 6(d-f). The water droplets stuck to the sand particles and stayed on the bare $\mathrm{Cu}$ surface, showing no self-cleaning effect. Through the selfcleaning experiments, the CuO-III surface with the hierarchical micro/nanostructure line array can be concluded to have great self-cleaning performance.
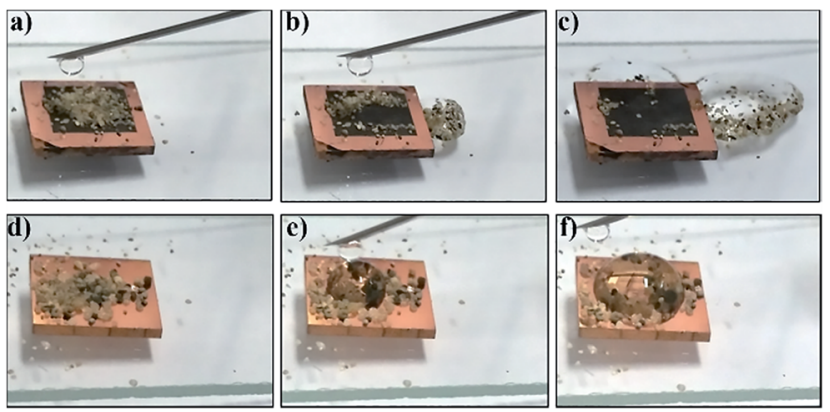

Fig. 6 Self-cleaning process on different surfaces: $(a-c)$ the line array structures fabricated by fs laser at the fluence of $0.60 \mathrm{~J} \mathrm{~cm}^{-2}$ after thermal oxidation and chemical modification, $(d-f)$ bare copper surface. 

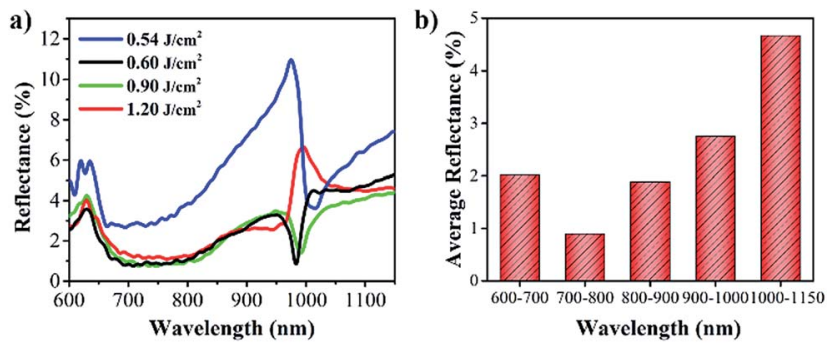

Fig. 7 (a) Reflectance of hierarchical micro/nanostructure line array surfaces fabricated at different laser fluences and under the same thermal oxidation condition. Polished $\mathrm{Cu}$ surface was set as the reference substrate with a reflectance of $100 \%$. (b) Average reflectance in different wavelengths ranges for hierarchical micro/nanostructure line array surfaces fabricated at a laser fluence of $0.60 \mathrm{~J} \mathrm{~cm}^{-2}$.

\section{Antireflection}

Micro/nanostructures have applications in antireflection, such as nanoneedles and nanocones. ${ }^{10}$ In this study, the hierarchical micro/nanostructure line arrays fabricated using different laser fluences exhibited high antireflective performance over a broad range of wavelengths $(600-1150 \mathrm{~nm})$, as illustrated in Fig. 7(a). The reflection spectra for the hierarchical micro/nanostructure line array surfaces decreased to less than $11 \%$ over a broad wavelength range. In particular, the $\mathrm{SiO}_{2}$ thin film was removed at a fluence of $0.54 \mathrm{~J} \mathrm{~cm}^{-2}$; thus, the microridges and nanoneedles were not sufficiently abundant to trap the light (Fig. 7(a)). As the laser fluence increased, the growth of microridges and nanoneedles significantly increased. The microridges and nanoneedles could impart stronger geometrical light trapping effects to the sample surfaces, thereby resulting in lower reflectance. ${ }^{17}$ Particularly, the $\mathrm{CuO}$ nanoneedles could induce phonon dissipation and eliminate the incident photons' energy through multi-internal reflection among the oxide nanoneedles while achieving a much lower overall surface reflectance. ${ }^{17}$ Therefore, the total reflectance of the sample considerably decreased to less than 6\% in 600-1150 $\mathrm{nm}$ range when the laser fluence slightly increased to $0.60 \mathrm{~J} \mathrm{~cm}^{-2}$. However, when the laser fluence was further increased, the reflectance of the samples hardly remained unchanged. Fig. 7(b) shows the average reflectance in different wavelength ranges for the hierarchical micro/nanostructure line array surfaces fabricated at the laser fluence of $0.60 \mathrm{~J} \mathrm{~cm}^{-2}$. It shows that the arithmetic average values of the measured reflectance in the 600-700 $\mathrm{nm}, 700-800 \mathrm{~nm}$, and 800-900 $\mathrm{nm}$ ranges were all lower than $2 \%$. In particular, the average reflectance decreased to less than $1 \%$ in the $700-800 \mathrm{~nm}$ range. Overall, the average reflectance was all lower than $5 \%$ in $600-1150 \mathrm{~nm}$ range.

\section{Conclusions}

In this study, hierarchical micro/nanostructure arrays with superhydrophobicity, self-cleaning and antireflection properties were fabricated by femtosecond laser ablation and thermal oxidation method. The morphologies of $\mathrm{CuO}$ micro-protrusions and the nanoneedles could be well tuned by controlling the femtosecond laser energy to obtain the desired surface properties. The line array surface could exhibit optimal superhydrophobicity with the contact angle and sliding angle of approximately $161^{\circ}$ and $1.7^{\circ}$, respectively, exhibiting excellent self-cleaning effect. Through the light trapping in the micro/ nanostructures, the reflectance could be less than $1 \%$ at the 700-800 nm wavelength range and keep steadily below 6\% over a broad wavelength range of 600-1150 $\mathrm{nm}$. Moreover, both the highly ordered micro-protrusions and the nanoneedles can be effectively reproduced, representing significant advantages for practical application. Besides, our approach may be suitable for processing large-area micro/nanostructures on various metallic materials.

\section{Conflicts of interest}

There are no conflicts to declare.

\section{Acknowledgements}

This work was supported by the National Natural Science Foundation of China (NSFC) (Grant No. 91323301 and 51675048), and the 863 of Ministry of Science and Technology of China (Grant No. 2015AA042702).

\section{Notes and references}

1 Z. Guo, X. Chen, J. Li, J. H. Liu and X. J. Huang, Langmuir, 2011, 27, 6193-6200.

2 L. Zhang, Y. Zhong, D. Cha and P. Wang, Sci. Rep., 2013, 3, 2326.

3 Q. X. Zhang, Y. X. Chen, Z. Guo, H. L. Liu, D. P. Wang and X. J. Huang, ACS Appl. Mater. Interfaces, 2013, 5, 1063310642.

4 F. Su and K. Yao, ACS Appl. Mater. Interfaces, 2014, 6, 87628770 .

5 A. Y. Vorobyev and C. Guo, J. Appl. Phys., 2015, 117, 033103. 6 X. Du, Y. Xing, X. Li, H. Huang, Z. Geng, J. He, Y. Wen and $\mathrm{X}$. Zhang, RSC Adv., 2016, 6, 7864-7871.

7 X. Gao, J. Zhou, R. Du, Z. Xie, S. Deng, R. Liu, Z. Liu and J. Zhang, Adv. Mater., 2016, 28, 168-173.

8 S. Ye, Q. Cao, Q. Wang, T. Wang and Q. Peng, Sci. Rep., 2016, 6, 37591.

9 W. Zhao, X. Liu, Y. Xu, S. Wang, T. Sun, S. Liu, X. Wu and Z. Xu, RSC Adv., 2016, 6, 35527-35531.

10 J. Zhu, Z. Yu, G. F. Burkhard, C.-M. Hsu, S. T. Connor, Y. Xu, Q. Wang, M. McGehee, S. Fan and Y. Cui, Nano Lett., 2008, 9, 279-282.

11 T. Hussain, L. Zhong, M. Danesh, H. Ye, Z. Liang, D. Xiao, C. W. Qiu, C. Lou, L. Chi and L. Jiang, Nanoscale, 2015, 7, 10350-10356.

12 J. Zhang, L. Zhong, Y. Sun, A. Li, J. Huang, F. Meng, B. K. Chandran, S. Li, L. Jiang and X. Chen, Adv. Mater., 2016, 28, 2978-2982.

13 Y. Li, X. Wei, B. Zhu, H. Wang, Y. Tang, T. C. Sum and X. Chen, Nanoscale, 2016, 8, 11284-11290. 
14 H. Yin, S. Zhao, K. Zhao, A. Muqsit, H. Tang, L. Chang, H. Zhao, Y. Gao and Z. Tang, Nat. Commun., 2015, 6, 6430.

15 N. Liu, R. Qu, Y. Chen, Y. Cao, W. Zhang, X. Lin, Y. Wei, L. Feng and L. Jiang, Nanoscale, 2016, 8, 18558-18564.

16 M. Zhao, K. Yuan, Y. Wang, G. Li, J. Guo, L. Gu, W. Hu, H. Zhao and Z. Tang, Nature, 2016, 539, 76-80.

17 P. Fan, B. Bai, J. Long, D. Jiang, G. Jin, H. Zhang and M. Zhong, Nano Lett., 2015, 15, 5988-5994.

$18 \mathrm{~J}$. Li, J. Zhu and X. Gao, Small, 2014, 10, 2578-2582.

19 X. Tan, L. Jiang, J. Hu, P. Liu, A. Wang and Y. Lu, Chin. Opt. Lett., 2015, 13, 111401-111404.

20 L. Jiang, Y. Sun, F. Huo, H. Zhang, L. Qin, S. Li and X. Chen, Nanoscale, 2012, 4, 66-75.

21 P. Yang, J. Zheng, Y. Xu, Q. Zhang and L. Jiang, Adv. Mater., 2016, 28, 10508-10517.

22 T. Guo, L. Heng, M. Wang, J. Wang and L. Jiang, Adv. Mater., 2016, 28, 8505-8510.

23 X. Hou, H. Zhang and L. Jiang, Angew. Chem., Int. Ed. Engl., 2012, 51, 5296-5307.

24 X. Gao and L. Jiang, Nature, 2004, 432, 36.

25 Y. Zheng, H. Bai, Z. Huang, X. Tian, F. Q. Nie, Y. Zhao, J. Zhai and L. Jiang, Nature, 2010, 463, 640-643.

26 S. Wan, H. Hu, J. Peng, Y. Li, Y. Fan, L. Jiang and Q. Cheng, Nanoscale, 2016, 8, 5649-5656.

27 F. Xia and L. Jiang, Adv. Mater., 2008, 20, 2842-2858.

28 L. Feng, S. Li, Y. Li, H. Li, L. Zhang, J. Zhai, Y. Song, B. Liu, L. Jiang and D. Zhu, Adv. Mater., 2002, 14, 1857-1860.

29 W. Barthlott and C. Neinhuis, Planta, 1997, 202, 1-8.

30 C. Neinhuis and W. Barthlott, Ann. Bot., 1997, 79, 667-677.

31 T. Darmanin, E. Taffin de Givenchy, S. Amigoni and F. Guittard, Adv. Mater., 2013, 25, 1378-1394.

32 M. Ma and R. M. Hill, Curr. Opin. Colloid Interface Sci., 2006, 11, 193-202.
33 X. Xu, Z. Zhang and J. Yang, Langmuir, 2010, 26, 3654-3658. 34 B. Bao, J. Sun, M. Gao, X. Zhang, L. Jiang and Y. Song, Nanoscale, 2016, 8, 9556-9562.

35 A. Wang, L. Jiang, X. Li, Y. Liu, X. Dong, L. Qu, X. Duan and Y. Lu, Adv. Mater., 2015, 27, 6238-6243.

36 A. Wang, L. Jiang, X. Li, Q. Xie, B. Li, Z. Wang, K. Du and Y. Lu, J. Mater. Chem. B, 2017, 5, 777-784.

37 S. Hermann, N.-P. Harder, R. Brendel, D. Herzog and H. Haferkamp, Appl. Phys. A: Mater. Sci. Process., 2009, 99, 151-158.

38 T. Rublack, S. Hartnauer, P. Kappe, C. Swiatkowski and G. Seifert, Appl. Phys. A: Mater. Sci. Process., 2011, 103, 43-50.

39 T. Rublack, M. Schade, M. Muchow, H. S. Leipner and G. Seifert, J. Appl. Phys., 2012, 112, 023521.

40 S. Rapp, M. Domke, M. Schmidt and H. P. Huber, Phys. Procedia, 2013, 41, 734-740.

41 S. Rapp, M. Schmidt and H. P. Huber, Appl. Phys. A: Mater. Sci. Process., 2016, 122, 1035.

42 X. Ji, L. Jiang, X. Li, W. Han, Y. Liu, Q. Huang and Y. Lu, Appl. Opt., 2014, 53, 6742-6748.

43 K. Lou, S. Qian, Z. Ren, X. Wang, Y. Li, C. Tu and H. Wang, J. Opt. Soc. Am. B, 2012, 29, 2282-2287.

44 M. Gecevičius, M. Beresna, J. Zhang, W. Yang, H. Takebe and P. G. Kazansky, Opt. Express, 2013, 21, 3959-3968.

45 X. C. Jiang, T. Herricks and Y. N. Xia, Nano Lett., 2002, 2, 1333-1338.

46 M. Kaur, K. P. Muthe, S. K. Despande, S. Choudhury, J. B. Singh, N. Verma, S. K. Gupta and J. V. Yakhmi, J. Cryst. Growth, 2006, 289, 670-675.

47 L. S. Huang, S. G. Yang, T. Li, B. X. Gu, Y. W. Du, Y. N. Lu and S. Z. Shi, J. Cryst. Growth, 2004, 260, 130-135.

48 C. H. Xu, C. H. Woo and S. Q. Shi, Chem. Phys. Lett., 2004, 399, 62-66. 